JKP-10 Japan-Korea Joint Session of the 92nd Annual Meeting of the Japanese Pharmacological Society

\title{
Adapalene induces adipose browning via RAR activation
}

\author{
Na Hyun Lee ${ }^{1}$, Hyae Gyeong Cheon ${ }^{1,2}$
}

${ }^{I}$ Department of Health Sciences and Technology,, ${ }^{2}$ Department of Pharmacology, College of Medicine, Gachon University, Incheon, 21999, Republic of Korea

Obesity is characterized by an excessive accumulation of fat and associated with various metabolic diseases. Recently, conversion of white adipocytes to brown-like adipocytes (beige adipocytes), naming browning has been focused as an attractive therapeutic strategy for the treatment of obesity. In the present study, we found that adapalene, a retinoic acid (RA) analogue that is often used as an anti-acne drug, induces adipose browning. Adapalene concentration dependently increased mRNA and protein expressions of browning markers including uncoupling protein-1 (UCP-1) in fully differentiated 3T3-L1 white adipocytes and in murine primary adipocytes. In agreement with increased expression of browning markers, reduced lipid droplets, and increased oxygen consumption rates and mitochondrial biogenesis were detected upon adapalene treatment. Conversely, knockdown of RARs by siRNAs attenuated adapalene-induced browning, suggesting that adapalene-induced browning is associated with RAR activation. In vivo browning effect of adapalene was confirmed using C57BL/6J mice. In summary, these data suggest that adpalene induces browning of white adipocytes via RAR activation, implying its possible new application as an anti-obesity drug. 